\title{
Study on the Correlation Between the Imbalance of Muscle Strength and the Score of EMG-Biofeedback Game at Ankle Joint in Healthy Adults
}

\author{
Yu-Min Ko' , Seol Park², Chang-Hun Lim¹, Woo-Jin Lee', Ji-Won Park² \\ 'Department of Physical Therapy, Gangneung Yeongdong College, Gangneung; ${ }^{2}$ Department of Physical Therapy, College of Medical Science, \\ Catholic University of Daegu, Daegu, Korea
}

Purpose: This study investigated whether the strength imbalance between two muscles can affect the score of EMG based biofeedback game, and whether the EMG based biofeedback game score can be used as predictable indicator of the degree of muscle balance alternating the conventional strength measuring equipment.

Methods: 40 participated in this study. Biodex was used to measure the peak torque/weight in order to calculate the muscle strength balance index between plantar flexor and dorsiflexor of ankle joint. And muscle balance index (MBI) was calculated. The EMG biofeedback game scores of dorsiflexor and plantar flexor were acquired, so that the EMG electrodes were attached at tibialis anterior and gastrocnemius. The relationship between the game score and the muscle balance index were analyzed.

Results: There was negative correlation between the muscle balance index between plantar flexor and dorsiflexor and the peak torque/ weight of plantar flexor $(r=-0.70)$. And there was negative correlation between the muscle balance index between plantar flexor and dorsiflexor and the game score of plantar flexor $(r=-0.83)$.

Conclusion: The EMG biofeedback game score had significant relationship with muscle imbalance at ankle joint, so it seems that the game score can be used for predicting the degree of muscle imbalance as a parameter.

Keywords: Electromyography, Games, Muscle strength

\section{INTRODUCTION}

As the development of highly information-oriented society, the portion using a computer has been increasing in daily life and computer can control almost devices being used on a daily basis. ${ }^{1}$ In addition, it is a trend that the interests about the human-computer interaction (HCI) is increasing as the alternative input way to use easily and conveniently for user and to overcome the limitation of previous input way such as a keyboard or a mouse (Kim 2005). In particular, the HCI is expected for elderly, physically disabled who loss normal motor ability, or mentally disabled who have difficulty in learning manipulation of machine to use as an equipment for communication or a controller of aids such as a wheel chair. ${ }^{2}$

The HCI is generally used to an inherent signal in the body such

Received Nov 14, 2015 Revised Dec 22, 2015

Accepted Dec 24, 2015

Corresponding author Ji-Won Park

E-mail mylovept@hanmail.net as voice, visual (gestures, facial expressions), biological signals (electroencephalogram, electromyogram, electrocardiogram or electrooculogram) as interface. Especially, the bio-signal interface may be used as the human-friendly and portable interface in wearable computing or mobile computing environment. In addition, the biosignal is used as not only HCI but also medical diagnosis, medical examination and healthcare through bio-signal analysis in the medical field..$^{3-6}$ So studies about the bio-signal are being conducted nowadays. $^{2}$

As the development of the Nintendo Wii that can be extensible as the functional games, sensible interfaces have begun to be highlighted in the gaming industry, the development of interface to utilize the various sensory has been enlarged. ${ }^{7-9}$ There is a report that these consoles can more improve the will of participation of reha-

Copylight (C2015 The Korea Society of Physical Therapy

This is an Open Access article distribute under the terms of the Creative Commons Attribution Non-commercial License (Http:// creativecommons.org/license/by-nc/3.o.) which permits unrestricted non-commercial use, distribution, and reproduction in any medium, provided the original work is properly cited. 
bilitation than a traditional way of treatment when they are applied to the patients for the purpose of rehabilitation. ${ }^{8,10}$ However, consoles commercially available for the public, are expected to have difficulty in rate and degree of difficulty of game interface section to apply to a patient. ${ }^{11}$ Moreover, most consoles such as the Nintendo Wii detect angles, three-dimensional motion, or acceleration as an interface, it is considered that the use for selective strengthening of specific paralyzed or weakened muscles or for performing movements without compensation will not be available.

Among various biofeedback for restoring the damaged motor function of the upper extremity in patients, EMG biofeedback is the most effective. ${ }^{12}$ The EMG biofeedback can be a key factor for improving the effectiveness of exercise therapy in patients by checking the statement of muscles and utilizing it at the training. ${ }^{4,13}$ The EMG biofeedback can be used for the rehabilitation using games as a medium rather than simple audiovisual auditory stimulation. In this case, it can lead patients to perform specific rehabilitation through fun games, to induce the exact exercise through EMG signals for the paralyzed or weakened muscles, and to enhance performance by facilitating motor learning through the use of bio-signals provided by the real-time biofeedback. ${ }^{14}$ In addition, by increasing the will to participate actively for patients and the satisfaction of the treatment, it is very important in increasing the effect of rehabilitation and in maintaining the rehabilitation activities. ${ }^{15,16}$

The EMG Biofeedback game is the game acquiring scores based on the ability to control properly through agonist contraction and antagonist relaxation for the intended movement. It may require the coordination between the agonist and the antagonist, proprioception as well as the muscular strength. In particular, a relative strength ratio between the agonist and the antagonist, that is, the ipsilateral muscle imbalance affects the physical performance. ${ }^{17}$ It may be the important factor to create the correct motion, and may affect to perform EMG-based game that utilizes the muscle activity. The ipsilateral muscle imbalance is the index indicating the degree of balanced development of the muscles around the joints, and it has been used as a good indicator to utilize the muscle strengthening, the injury prevention, the rehabilitation programs after surgery for the public as well as athletes. ${ }^{18,19}$ People with chronic joint instability have the ipsilateral muscle imbalance, and especially, ankle joint is the most commonly injured with a relatively weak structure, which can cause joint instability. ${ }^{20,21}$
Therefore, this study investigated whether the strength imbalance between two muscles can affect the score of EMG based biofeedback game, and whether the EMG based biofeedback game score can be used as predictable indicator of the degree of muscle balance alternating the conventional strength measuring equipment.

\section{METHODS}

\section{Subjects}

40 (mean age $22.8 \pm 2.3$ years, height $166.9 \pm 7.6 \mathrm{~cm}$, weight $56.9 \pm 9.8$ $\mathrm{kg}$, BMI $20.3 \pm 2.2 \%$ ) participated in this study. Subjects who were selected had no any congenital deformities on his knees and ankles, and no neurological and orthopedic diseases during the last 6 months. Their height and body weight were measured before the experiments in order to know the general characteristics of subjects. 12 males (mean age $25.1 \pm 2.0$ years, height $176.3 \pm 4.1 \mathrm{~cm}$, body weight $69.1 \pm$ $6.6 \mathrm{~kg}$, BMI $22.3 \pm 2.2 \%$ ) and 28 females (mean age 21.81.7 years, height $162.8 \pm 4.5 \mathrm{~cm}$, body weight $51.6 \pm 5.0 \mathrm{~kg}$, BMI $19.5 \pm 1.5 \%$ ) participated. Subjects agreed voluntarily to participate in this experiment after listening to the detailed explanation about this study.

\section{Experimental methods}

\section{1) Muscle Balance Index (MBI)}

Biodex system 4 Pro Dynamometer and Biodex Advantage Software Package (Biodex Medical Systems Inc., Shirley, USA) were used to measure the isokinetic concentric maximal strength in order to calculate the muscle strength balance index between plantar flexor and dorsiflexor of ankle joint. The measured leg was fixed on the rest $40 \mathrm{~cm}$ height using straps while lying fully backward. All subjects wore the same shoes, and the axis of their ankle joint and dynamometer were arranged. The non-measured leg was fixed with 90 degree flexed knee, and the foot was on the rest. The maximal strength was calculated by dividing the peak torque by the body weight at $60 \% \mathrm{sec}$ angular velocity. It was measured 5 times while subjects exerted their greatest capability with consistent verbal commands. The muscle strength balance index (MBI) was calculated as following: the higher value of MBI, the larger degree of the muscle imbalance between two muscles.

$\mathrm{MBI}=\left|\frac{\text { Strength of dorsiflexor-Strength of plantar flexor }}{\text { Strength of dorsiflexor }+ \text { Strength of plantar flexor }}\right|$ 


\section{2) EMG biofeedback game score}

The EMG electrodes were attached at tibialis anterior and calf in order to acquire game scores of EMG biofeedback game system. The electrodes for tibialis anterior, the dorsiflexor, were attached to the outside of the anterior tibia region $2 \mathrm{~cm}$, at the position laterally $2 \mathrm{~cm}$ from the anterior line of tibia, and the electrodes for calf muscle, the plantar flexor, were attached to the medial surface of a line $2 \mathrm{~cm}$ away from the popliteal part centerline. ${ }^{22}$ Then, the muscle strength balance index of individual muscles was calibrated in order to consider the individual differences.

After attaching the EMG electrodes at each muscles, subjects started the game. They played three times, and the average value was used. And the relationship between the acquired game score and the MBI of two muscles was analyzed.

When the EMG signal of a muscle is over than specific threshold though contracting the muscle, the computer converts the EMG signal to the specific direction key. It makes the game character move in the direction, and the game score is obtained when the game character touches some icons by contracting each muscles corresponding each directions.

According to the EMG signal obtained by each muscle contraction, the game character could be moved up or down. It was designed that various types of fish were to be passed from the right to left hand side of the screen. A point was acquired when the game character made contact with a passing fish, while the game was over when the character could not contact a fish three times.

The game scores and muscle strength of plantar flexor and dorsiflexor at ankle joint were measured, and the MBI was calculated. Then, we investigated whether the MBI and the game score have a correlation.

\section{3) Statistical analysis}

SPSS ver. 18.0 for Windows was used for statistical analysis. Pearson correlation and regression were used for analyzing the relationship between the game score and the MBI. Significance level was $\mathrm{p}<0.05$.

\section{RESULTS}

\section{Relationship between the peak torque/weight of plantar flexor and dorsiflexor and the MBI}

There was negative correlation between the MBI and the peak torque/weight of plantar flexor $(r=-0.70)$, and it indicates that the larger peak torque/weight of plantar flexor, the lower muscle imbalance. In the other hand, the peak torque/weight of dorsiflexor had no effect on muscle imbalance $(r=-0.06)$ (Table 1$)$.

Table 1. The correlation between balance of peak torque/body weight between plantar flexor and dorsiflexor and game score by ankle plantar flexor and dorsiflexor

\begin{tabular}{lccccc}
\hline & SPF & SDF & tPF & tDF & MBI \\
\hline Value $(\mathrm{M} \pm$ SD) & $192.37 \pm 45.53$ & $220.53 \pm 20.26$ & $27.52 \pm 22.08$ & $17.58 \pm 20.26$ & $0.13 \pm 0.24$ \\
SPF & 1 & 0.30 & $0.58^{*}$ & 0.05 & $-0.83 *$ \\
SDF & & 1 & 0.28 & 0.25 & -0.11 \\
tPF & & & 1 & $0.65^{*}$ & $-0.70^{*}$ \\
tDF & & & & 1 & -0.06 \\
MBI & & & & 1 \\
\hline
\end{tabular}

sPF: Game score by plantar flexor in ankle joint, sDF: Game score by dorsiflexor in ankle joint, tPF: Peak torque/body weight of plantar flexor in ankle joint, tDF: Peak torque/body weight of dorsiflexor in ankle joint, MBI: Dorsiflexor strength-plantar flexor strength/Dorsiflexor strength+plantar flexor strength.

*Statistically significant at the level of $p<0.01$

Table 2. The results of regression between game score of plantar flexor and balance of peak torque/body weight between plantar flexor and dorsiflexor in ankle joint

\begin{tabular}{|c|c|c|c|c|c|c|c|}
\hline & & $\mathrm{R}^{2}$ & $B(\beta)$ & SE & $\mathrm{F}$ & $\mathrm{t}$ & $p$ \\
\hline \multirow[t]{2}{*}{ sDF } & Constant & & 46.42 & 79.25 & & 0.59 & 0.56 \\
\hline & sDF & 0.08 & $0.66(0.30)$ & 0.36 & 3.42 & 1.85 & 0.07 \\
\hline \multirow[t]{2}{*}{$\mathrm{MBI}$} & Constant & & 213.12 & 4.79 & & 44.48 & 0.00 \\
\hline & $\mathrm{MBI}$ & 0.69 & $-158.31(-0.83)$ & 17.79 & 79.18 & -8.90 & $0.00^{*}$ \\
\hline
\end{tabular}

sDF: Game score by dorsiflexor in ankle joint, MBI: Balance of peak torque/Body weight between plantar flexor and dorsiflexor in ankle joint.

*Statistically significant at the level of $p<0.01$. 


\section{Relationship between the MBI and the game scores of plantar}

\section{flexor and dorsiflexor}

There was negative correlation between the MBI and the game score of plantar flexor $(\mathrm{r}=-0.83)$ (Table 1). It indicates that the larger muscle imbalance, the lower game score of plantar flexor $(\mathrm{p}<0.01)$ (Table 2). According to the regression analysis, the sDF cannot predict $\mathrm{SPF}\left(\mathrm{R}^{2}=0.08\right)$, but the MBI can predict $69 \%$ of the $\mathrm{PPF}$. The regression equation is as following;

$$
\mathrm{sPF}=213.12+(-158.31) \times \mathrm{MBI}
$$

In the other hand, the MBI had no effect on the game score of dorsiflexor $(\mathrm{r}=-0.11, \mathrm{p}>0.05)$ (Table 3). According to the regression analysis, the $\mathrm{sPF}$ and MBI cannot predict $\mathrm{sDF}\left(\mathrm{R}^{2}=0.09,0.01\right.$, respectively) (Figure 1).

\section{DISCUSSION}

In this study, EMG based physical interactive game was developed in order to induce patients' active participation through overcoming the boredom and monotony of the rehabilitation that is performed for a long time. And this study investigated whether the game score has a correlation with muscle strength and ipsilateral MBI that were measured by conventional isokinetic strength measuring equipment.

There was positive relationship between the peak torque/weight of plantar flexor and those dorsiflexor $(r=0.65)$, so subjects who had stronger strength of plantar flexor had stronger strength of dorsiflexor (Table 1). And subjects with larger strength of plantar flexor had lower muscle imbalance $(r=-0.70)$. The MBI was calculated to be (dorsiflexor strength-plantar flexor strength)/(dorsiflexor strength+plantar flexor strength). It was predictable that subjects with stronger strength of dorsiflexor had lower muscle imbalance, but strength of dorsiflexor had no effect on MBI unlike those plantar flexor $(r=-0.06)$. It may be because the peak torque/weight of plantar flexor $(27.52 \pm 22.08)$ was larger than those dorsiflexor (17.58 \pm 20.26 ), so the MBI might have a greater effect on the plantar flexor strength. And the dorsiflexor strength might have lesser influence on the degree of muscle imbalance than plantarflexor strength, because the standard deviation of the peak torque/weight of dorsiflexor was small against those of plantar flexor. Therefore, the muscle imbalance between plantar flexor and dorsiflexor may depend on the plantar flexor strength.

The peak torque/weight of dorsiflexor was lesser than those of plantar flexor, but the game score of dorsiflexor was higher than those of plantar flexor (sPF : 192.37 \pm 45.53 , sDF : $220.53 \pm 20.26$ ). Dorsiflexors establish the foot position by the statement of ground surface during landing the foot, and especially, determine the degree of ankle flexion while obstacle crossing or stair stepping. So they may control detail movements at ankle joint. It might induce the smooth movement of the game character, and they could acquire higher scores than plantar flexors.

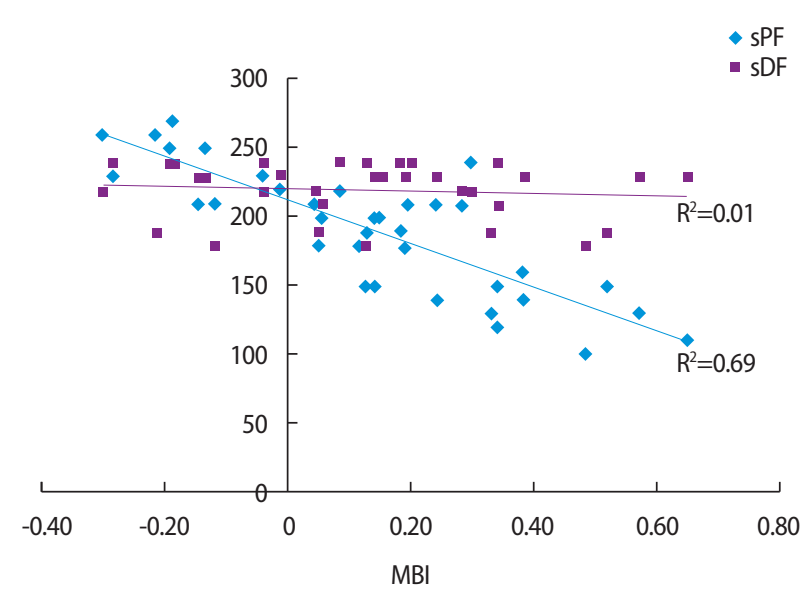

Figure 1. The correlation between the balance of the peak torque/ body weight between plantar flexor and dorsiflexor and obtained game score by plantar flexor and dorsiflexor.

MBI: Balance of peak torque/Body weight between plantar flexor and dorsiflexor, sPF: Obtained game score by plantar flexor, sDF: Obtained game score by dorsiflexor.

Table 3. The results of regression between game score of dorsiflexor and balance of peak torque/body weight between plantar flexor and dorsiflexor in ankle joint

\begin{tabular}{|c|c|c|c|c|c|c|c|}
\hline & & $\mathrm{R}^{2}$ & $B(\beta)$ & SE & $\mathrm{F}$ & $\mathrm{t}$ & $p$ \\
\hline \multirow[t]{2}{*}{ sDF } & Constant & & 195.31 & 14.00 & & 13.95 & 0.00 \\
\hline & sDF & 0.09 & $0.13(0.30)$ & 0.07 & 3.472 & 1.852 & 0.07 \\
\hline \multirow[t]{2}{*}{$\mathrm{MBI}$} & Constant & & 221.70 & 3.79 & & 58.45 & 0.00 \\
\hline & $\mathrm{MBI}$ & 0.01 & $-8.95(-0.11)$ & 14.09 & 0.40 & -0.64 & 0.53 \\
\hline
\end{tabular}

sPF: Game score by plantar flexor in ankle joint, MBI: Balance of peak torque/Body weight between plantar flexor and dorsiflexor in ankle joint.

*Statistically significant at the level of $p<0.01$. 
The larger muscle imbalance at ankle joint, the lower game score of plantar flexor $(\mathrm{r}=-0.83)$ while the muscle imbalance had no effect on the game score of dorsiflexor $(\mathrm{r}=-0.11)$. The MBI might affect the game score of plantar flexor $(\mathrm{r}=-0.83)$. The possible reasons are as following: First, the MBI was influenced on the plantar flexor strength $(r=-0.70)$ and subjects with stronger plantar flexor got higher game score $(r=0.58)$. In other words, the muscle imbalance may depend on the peak torque/weight of plantar flexor, and it may affect the game score of plantar flexor. Second, the game scores of dorsiflexor were generally high regardless of the degree of the muscle imbalance, so game score of dorsiflexor was not related to the muscle imbalance.

Dorsiflexors control detail movements at ankle joint, while plantar flexors are more powerful as they lift one's body such as heel raising. For this reason, the minimize threshold for activating plantar flexors may higher than those dorsiflexors. Game score of plantar flexor was measured during open kinetic chain exercise, and it might be difficult to activate over than the threshold for moving the game character through ankle plantar flexion during open kinetic chain. It may be the reason of lower game score of plantar flexor. If the game score was measured while raising heel in a vertical position, that is, closed kinetic chain exercise, it would generate enough activation over than threshold so that the game score of plantar flexor would be higher. However, in this case, it might be impossible to acquire higher score because subjects could feel fatigue easily or not control rapidly in order to control the game character through frequent heel raising.

In contrast, MBI had no effect on the game score of dorsiflexor $(\mathrm{r}=-0.11)$. In order to get high score, detailed control at ankle joint may be necessary in order to move the game character properly, and dorsiflexor may play a role in control delicately at ankle joint. Thus, subjects gained high scores of the game for dorsiflexor, and there was lesser differences of the game score of dorsiflexor between subjects $(220.53 \pm 20.26)$ than those of plantar flexor. In other words, even people with larger muscle imbalance gained high score of the game for dorsiflexor, and the MBI did not affect the game score of dorsiflexor. In this study, all subjects had not had any muscle injury or ankle pain, so it might affect the result that their scores of dorsiflexor were high. When people with ankle instability or pain had the decline of dorsiflexor strength and position sense. ${ }^{20}$ It is expected that ankle instability or pain can affect the game score, so future study should investigate the effects of the muscle strength or position sense in people with ankle instability on the game score.

In conclusion, the peak torque/weight of plantar flexor was larger than those of dorsiflexor, while the game score of plantar flexor was lower than those of dorsiflexor. In addition, muscle imbalance at ankle joint had an effect on the plantar flexor strength, not the dorsiflexor strength, and the game score had also an effect on the plantar flexor. Thus, in order to predict the muscle imbalance at ankle joint without any injury or pain, the game score of plantar flexor can be used as people with lower game score of plantar flexor had weaker peak torque/weight of plantar flexor.

Sports injuries occur when the strength of weak parts in body cannot withstand resistance during sports, and its muscles break away from the path. In general, when agonists are strong for movement, the antagonists get lots of stress as a stabilizer of the joint, and it can generate injuries at the joint if the antagonists are weak. So, it is important for antagonists or weakened muscles to strengthen in order to prevent injuries and improve functions, and it can maintain good posture by efficiently arranging the parts in body. ${ }^{23} \mathrm{Ac}$ cording to the results of this study, the game score had significant relationship with muscle imbalance, so it seems that the game score can be used for predicting the degree of muscle imbalance as a parameter.

The limitations in this study were as following: first, there were no control groups, because this study investigated the relationship between the degree of the muscle strength and game score. Second, the game system was not used as an intervention.

Future studies should study the correlation between the game score and proprioception that causes joint instability. Also, studies that verify the effects of the game system as therapeutic equipment for healthy adults or patients will be required.

\section{REFERENCES}

1. Kim JS, Kim HK, Jeong H et al. Human-computer interface based on bio-signal. Electronics and Telecommunications Trends. 2005;20(4):6781.

2. Lee AR, Ryu HK. Rehabilitation program design for the stroke patients and caregivers with Activity Theory. Journal of the HCI Society of Korea. 2013;2013(1):192-5

3. Kuk EJ, Kim JM. An EEG-based brain mapping to determine mirror neuron system in patients with chronic stroke during action observation. J Kor Phys Ther. 2015;27(3):135-9. 
4. Park SK, Kang JY. Effects of EMG-biofeedback training on functional ability and Q-angle in patellofemoral pain syndrome. J Kor Phys Ther. 2014;26(2):68-73.

5. Kim SJ. Effects of manual lymph drainage on the activity of sympathetic nervous system, anxiety, pain, and pressure pain threshold in subjects with psychological stress. J Kor Phys Ther. 2014;26(6):391-7.

6. Lim JH, Park JS, Cho WS. The effect of mechanical horseback-riding training velocity on vestibular functions and static postural balance in healthy adults. J Kor Phys Ther. 2013;25(5):288-96.

7. Lanningham-Foster L, Foster RC, McCrady SK et al. Activity-promoting video games and increased energy expenditure. J Pediatr. 2009;154(6): 819-23.

8. Lee GH. Effects of virtual reality exercise program on balance in multiple sclerosis patients. J Kor Phys Ther. 2015;27(1):61-7.

9. Park SD, Kim JY, Yu SH et al. Comparison of balance and fall efficacy of virtual reality program in elderly women with fall experience. J Kor Phys Ther. 2014;26(6):430-5.

10. Deutsch JE, Borbely M, Filler J et al. Use of a low-cost, commercially available gaming console (wii) for rehabilitation of an adolescent with cerebral palsy. Phys Ther. 2008;88(10):1196-207.

11. Anderson F, Annett M, Bischof WF. Lean on wii: Physical rehabilitation with virtual reality wii peripherals. Stud Health Technol Inform. 2010;154:229-34.

12. van Dijk H, Jannink MJ, Hermens HJ. Effect of augmented feedback on motor function of the affected upper extremity in rehabilitation patients: A systematic review of randomized controlled trials. J Rehabil Med. 2005;37(4):202-11.

13. Eleanor C. Cram's introduction to surface electromyography. 2nd Edi- tion. 2005:5-7.

14. Mulder T, Hulstyn W. Sensory feedback therapy and theoretical knowledge of motor control and learning. Am J Phys Med. 1984;63(5):226-44.

15. Lee DY. A study on the vocational rehabilitation facilities: Focusing on the current situations, conditions and administrative efficiency. Korean Journal of Physical, Multiple, \& Health Disabilities. 2010;53(1):1-24.

16. Jang JY, Yoon HS. A study on serious game design for rehabilitation training of stroke patients. Journal of The Korean Society for Computer Game. 2008;15:151-9.

17. Park CG. The effect of SAQ training for athletic performance and physical fitness in female basketball athletes. Journal of Coaching Development. 2010;12(2):219-28.

18. Sohn JH. The effect of muscle strength imbalance on motion of squat. Seoul National University. Dissertation of Master's Degree. 2009.

19. Fleck SJ, Falkel JE. Value of resistance training for the reduction of sports injuries. Sports Med. 1986;3(1):61-8.

20. Willems T, Witvrouw E, Verstuyft J et al. Proprioception and muscle strength in subjects with a history of ankle sprains and chronic instability. J Athl Train. 2002;37(4):487-93.

21. Choi HS, Shin WS, Shim JK et al. The relationship between functional movement screen and ankle dysfunctions with chronic ankle instability. J Kor Phys Ther. 2014;26(6):459-63.

22. Schulthies SS, Ricard MD, Alexander KJ et al. An electromyographic investigation of 4 elastic-tubing closed kinetic chain exercises after anterior cruciate ligament reconstruction. J Athl Train. 1998;33(4):328-35.

23. Jung IG, Kim SY. A comparative study of upper body's muscle power ratio between judo players and gymnastic players. Journal of Korea Sport Research. 2004;15(2):1383-90. 